\title{
Fatores determinantes para o desempenho em inovação das indústrias optantes lucro real em Santa Catarina
}

\section{Paloma Zimmer}

Doutoranda em Engenharia e Gestão do Conhecimento - Universidade Federal de Santa Catarina.Mestre em Engenharia e Gestão do Conhecimento - Universidade Federal de Santa Catarina.Bacharel em Comunicação Social com Habilitação em Publicidade e Propaganda - Fundação Universidade Regional de Blumenau.Programa de Pós-Graduação em Engenharia e Gestão do Conhecimento Centro Tecnológico (CTC) - Universidade Federal de Santa Catarina (UFSC),Santa Catarina, Brasil

palomazimmer@yahoo.com.br

Cristiane Mitsuê lata

Doutoranda em Engenharia e Gestão do Conhecimento - Universidade Federal de Santa Catarina. Mestre em Engenharia Mecânica - Universidade Federal de Santa Catarina Engenheira Eletricista - Centro Federal de Educação Tecnológica de Minas Gerais Programa de Pós-Graduação em Engenharia e Gestão do Conhecimento Centro Tecnológico (CTC) - Universidade Federal de Santa Catarina (UFSC), Santa Catarina, Brasil

cristianeiata@hotmail.com

João Artur de Souza

Doutor em Engenharia de Produção - Universidade Federal de Santa Catarina. Mestre em Matemática e Computação Científica - Universidade Federal de Santa Catarina.Graduado em Direito - Universidade do Sul de Santa Catarina.Graduado em Matemática Universidade Federal de Santa Catarina.Programa de Pós-Graduação em Engenharia e Gestão do Conhecimento.Centro Tecnológico (CTC) - Universidade Federal de Santa Catarina (UFSC), Santa Catarina, Brasil

jartur@gmail.com

Cristiano José Castro de Almeida Cunha

Doutor em Administração de Empresas - Rheinisch-Westfalische Technische Hochschule Aachen, RWTH, Alemanha.Mestre em Engenharia de Produção - Universidade Federal de Santa Catarina.Graduado em Engenharia Geológica - Universidade Federal de Ouro Preto Programa de Pós-Graduação em Engenharia e Gestão do Conhecimento Centro Tecnológico (CTC) - Universidade Federal de Santa Catarina (UFSC), Santa Catarina ,Brasil 01cunha@gmail.com

Editor Científico: José Edson Lara
Organização Comitê Científico
Double Blind Review pelo SEER/OJS
Recebido em 10.01.2016
Aprovado em 21.11.2017




\title{
Resumo
}

Este artigo tem como objetivo apresentar as principais práticas e entraves para a inovação e relacioná-las com o desempenho obtido em inovação em um grupo de 100 indústrias com matriz no estado de Santa Catarina, optantes pelo regime tributário do Lucro Real. Para isso, utilizou-se como estratégia de pesquisa um survey e, para a análise dos dados, estatística bi e multivariada. Esta pesquisa se caracteriza como descritiva e de abordagem quantitativa. Os resultados do estudo comprovam que a performance em inovação é resultado das práticas adotadas pela empresa, que vão desde a busca por novas matérias primas e processos produtivos, até o relacionamento de cooperação com fornecedores e concorrentes. Todas essas práticas tornam-se mais fluidas e efetivas para a organização, quando a mesma possui uma área formalizada para cuidar dos assuntos relacionados à Pesquisa, Desenvolvimento e Inovação - PD\&l.

Palavras-chave: Gestão da Inovação; barreiras para a inovação; práticas para inovação.

\section{Determining Factors for Innovation Performance from Industries in Santa Catarina State}

\begin{abstract}
This article presents the relations between practices and challenges to innovation. Literature in this area has shown that the main obstacles to innovation are economics aspects, such as risks and low incentives from Government. Organizational culture has not been mentioned as a principal challenge to innovation. The aim of this study is to analyze if aspects of organizational culture are overcome, and verify what aspects are related to the performance of innovation. To do it, was conducted a quantitative study with 100 industries from Santa Catarina State - Brazil. The results show that the performance in innovation is a result of organizational practices, and the companies do not introduce these practices in their routines.
\end{abstract}

Keywords: Innovation Management; challenges to innovation; innovation.

\section{Factores Determinantes para el Desempeño en Innovación de las Industrias optando Lucro Real en Santa Catarina}

\section{Resumen}

Este artículo tiene como objetivo presentar las principales prácticas y obstáculos para la innovación y relacionarlos con el desempeño obtenido en innovación en un grupo de 100 industrias con matriz en el estado de Santa Catarina - Brazil, optando por el régimen tributario del Beneficio Real. Para ello, se utilizó como estrategia de investigación un survey y, para el análisis de los datos, estadística bi y multivariada. Esta investigación se caracteriza como descriptiva y de abordaje cuantitativo. Los resultados del estudio demuestran que la performance en innovación es el resultado de las prácticas adoptadas por la empresa, que van desde la búsqueda de nuevas 
materias primas y procesos productivos, hasta la relación de cooperación con proveedores y competidores. Todas estas prácticas se vuelven más fluidas y efectivas para la organización, cuando la misma posee un área formalizada para cuidar de los asuntos relacionados a la Investigación, Desarrollo e Innovación PD\&I.

Palabras clave: gestión de la innovación; barreras para una innovación; prácticas para la innovación.

\section{Introdução}

Apesar do tema inovação já fazer parte do vocabulário dos empresários, o que se observa na prática são empresas com reduzidas taxas de inovação. Segundo o Global Entrepreneurship Monitor - GEM (2013), no Brasil, os novos empreendimentos possuem baixo potencial inovador, concentrando-se em atividades com reduzido conteúdo tecnológico, com pequenas barreiras de entrada, voltados somente para o mercado interno ou para a prestação de serviços ao consumidor. De acordo com o relatório, apenas 0,3\% dos empreendimentos apresentam alguma novidade.

Terra (2000) já sinalizava que o principal desafio para a inovação era a criação de uma equipe em que a informação e o conhecimento fluíssem rapidamente. Dessa forma, o compartilhamento de visões e conhecimentos a serem explorados pela empresa ocorreria de forma mais tranquila, resultando em vantagem competitiva.

Dados do IBGE (2013) apontam que os principais obstáculos para a inovação, segundo a percepção das empresas, são os riscos econômicos excessivos, a escassez de fontes apropriadas de financiamento e a fraca resposta dos consumidores a novos produtos. Ou seja, fatores internos à empresa não entram na relação dos três principais entraves para a inovação. Mas será que, de fato, os fatores internos já foram superados? As empresas possuem práticas de inovação que permeiam toda a organização e a resposta para o reduzido potencial de inovação das empresas brasileiras está na carência de recursos financeiros?

Este artigo tem como objetivo responder estes questionamentos, apresentando os fatores determinantes para o desempenho em inovação a partir das práticas adotadas por 100 indústrias com matriz no estado de Santa Catarina optantes pelo regime tributário do Lucro Real. O intuito é verificar quais ações que, de fato, reduzem os obstáculos e promovem a inovação. 
As barreiras para a inovação são objeto de estudo por diferentes autores. Feldens, Maccari e Garcez (2012) analisaram os principais obstáculos para a inovação em pequenas e médias empresas de base tecnológica. Ziviani e Ferreira (2013) avaliaram os fatores que dificultam a atividade de inovação no setor elétrico brasileiro. Kühl e Cunha (2013) estudaram como diferentes empresas percebem os obstáculos para a implementação de inovações. Os autores utilizaram como fonte de dados a Pesquisa de Inovação da PINTEC.

No entanto, a literatura que trata sobre este assunto analisa somente as barreiras percebidas para a inovação, e não as relacionadas com as práticas adotadas pelas empresas. Este artigo visa aclarar este gap da literatura.

A análise sobre os fatores que dificultam a realização de atividades de pesquisa e desenvolvimento, seja em âmbito regional ou setorial, torna-se relevante tendo-se em vista que permite o desenvolvimento de estratégias que visem promover a inovação alinhada às necessidades diagnosticadas.

Neste artigo optou-se pela realização de um estudo regional. Os resultados retratam as práticas e desafios para a inovação das empresas sediadas em Santa Catarina. $O$ artigo está dividido em cinco seções, sendo a primeira delas esta introdução. A segunda apresenta o referencial teórico, abordando questões relacionadas à inovação. Na terceira seção apresentam-se os procedimentos metodológicos utilizados na pesquisa que deu origem aos dados deste artigo. $\mathrm{Na}$ quarta seção são apresentados os principais entraves para a inovação. $O$ artigo é concluído na quinta seção com as considerações finais.

\section{Inovação: Conceitos e Definições}

A inovação é o que promove o crescimento econômico no longo prazo e assegura a competitividade da empresa. Para Schumpeter (1988, p. 76), a inovação tecnológica cria uma ruptura no sistema econômico, alterando padrões de produção e proporcionando diferenciação para a empresa.

A definição de inovação utilizada no Manual de Oslo (2005, p.55 §146) é mais branda quanto à ruptura em sistemas econômicos. O impacto da inovação pode ser apenas para a empresa e não para o mercado. Pois, de fato, o Manual de Oslo tem o objetivo de orientar e, principalmente, padronizar conceitos e metodologias, a fim de proporcionar uma linguagem comum, visando à construção de estatísticas e indicadores de pesquisa de PD\&I para países industrializados e membros da OCDE. 
De acordo com o Manual de Oslo (2005, §80), na visão de Schumpeter a inovação é um aspecto da estratégia de negócios ou uma parte do conjunto de decisões de investimentos para criar capacidade de desenvolvimento de produto ou para melhorar a eficiência da empresa. A inovação deve provocar mudanças amplas e extensivas, que reestruturem indústrias e mercados.

Para Canto (apresentação edição Manual Oslo, 2005), como o Manual de Oslo é bastante abrangente e flexível quanto às suas definições e metodologias de inovação tecnológica, ele tem sido uma das principais referências para as atividades de inovação nas indústrias brasileiras.

No Brasil, a Lei 10.973/2004 (art. 2o IV), conhecida como Lei de Inovação, estabelece medidas de incentivo à inovação e à pesquisa científica e tecnológica no ambiente produtivo. O conceito de inovação definido nesta lei é a "introdução de novidade ou aperfeiçoamento no ambiente produtivo e social que resulte em novos produtos, serviços ou processos ou que compreenda a agregação de novas funcionalidades ou características a produto, serviço ou processo já existente que possa resultar em melhorias e em efetivo ganho de qualidade ou desempenho".

Para Drucker (2013), inovação envolve valor econômico. Segundo o autor, inovação é a capacidade de criar riqueza por meio de recursos. O recurso não existe até que o homem encontre um uso para ele e assim o dote de valor econômico.

Tidd, Bessant e Pavitt (2008) afirmam que a inovação não é algo estático, e nem sempre está relacionada a algo que já faça parte das competências de uma empresa. Neste caso a inovação envolve assumir riscos e explorar recursos normalmente escassos em projetos que podem muito bem fracassar.

Para os autores, a inovação está atrelada ao conhecimento, já que ela é resultado da combinação entre diferentes conjuntos de conhecimentos. Esses níveis de conhecimentos dependem do que já foi vivenciado, baseado em experiência passadas, ou é algo relacionado à busca por respostas, sejam elas tecnológicas, de mercado, concorrência, dentre outras.

O Manual de Oslo (2005) define quatro tipos de inovações, todos alicerçados em um amplo conjunto de mudanças nas atividades das empresas. Essas inovações podem ser: (i) de produto; (ii) em processo; (iii) organizacionais; e (iv) inovações de marketing. 
$\mathrm{Na}$ Tabela 1 são apresentadas as classificações dos tipos de inovação quanto à natureza com suas respectivas descrições.

\section{Tabela 1}

Classificação da inovação quanto à natureza

\begin{tabular}{l|l}
\hline Natureza & Descrição \\
\hline Produto & $\begin{array}{l}\text { As inovações de produto envolvem mudanças significativas nas } \\
\text { potencialidades de produtos e serviços. Incluem-se bens e serviços } \\
\text { totalmente novos e aperfeiçoamentos importantes para produtos } \\
\text { existentes. }\end{array}$ \\
\hline Processo & $\begin{array}{l}\text { As inovações de processo representam mudanças significativas nos } \\
\text { métodos de produção e de distribuição. A empresa pode realizar vários } \\
\text { tipos de mudanças em seus métodos de trabalho, seu uso de fatores de } \\
\text { produção e os tipos de resultados que aumentam sua produtividade e/ou } \\
\text { seu desempenho comercial. }\end{array}$ \\
\hline Organizacional & $\begin{array}{l}\text { As inovações organizacionais referem-se à implementação de novos } \\
\text { métodos organizacionais, tais como: mudanças em práticas de negócios, } \\
\text { na organização do local de trabalho ou nas relações externas da } \\
\text { empresa. }\end{array}$ \\
\hline Marketing & $\begin{array}{l}\text { As inovações de marketing envolvem a implementação de novos métodos } \\
\text { de marketing, incluindo mudanças no "design" do produto e na } \\
\text { embalagem, na promoção do produto e sua colocação, e em métodos de } \\
\text { estabelecimento de preços de bens e de serviços. }\end{array}$ \\
\hline
\end{tabular}

Fonte: Adaptado de Oslo (2005).

Além da distinção quanto à natureza, a inovação pode ser classificada, segundo Mattos, Stoffel e Teixeira (2010), quanto à forma (tecnológica ou organizacional); à abrangência (na empresa, no mercado ou no mundo); à intensidade (incremental, semirradical e radical); ao propósito (ocasional ou intencional/sistemática); ao nível de difusão dentro da empresa (localizada/departamental ou sistêmica); e, finalmente, quanto ao uso de sistemas, métodos e ferramentas podendo ser empírica ou sistemática/metodológica.

Tidd, Bessant e Pavitt (2008) atribuem quatro classificações para a inovação: (I) Inovação incremental: as regras do jogo já estão claras, consiste no incremento estável de produtos ou processos e no uso de conhecimento acumulado acerca de componentes centrais do que atualmente já faz parte do core business da empresa; (II) Inovação modular: são realizadas alterações significativas em apenas um elemento, porém a arquitetura geral do produto ou processo permanece a mesma. A aprendizagem de conhecimento está restrita somente a um componente, com fontes de conhecimentos e usuários bem definidos. As melhorias existem dentro do parâmetro "fazer o que sabemos, mas melhor"; (III) Inovação de arquitetura: novas combinações, o que Tidd, Bessant e Pavitt (2008) definem como arquiteturas, surgem. Os desafios para este tipo de inovação consistem em remodelar as fontes de conhecimento. Pode-se abrir mão dos conhecimentos já existentes ou recombiná- 
los de diferentes formas, incluindo novos conhecimentos neste processo; (IV) Inovação descontínua: tanto o produto final quanto os processos são plenamente conhecidos. Todo o conjunto de regras é alterado e abre-se espaço para novos entrantes.

Apesar de existirem diferentes classificações para a inovação, o que se observa, é que, no Brasil, as empresas lançam-se no mercado com um produto inovador, porém com o passar dos anos o que era inovador torna-se obsoleto. Nesse caso, ou a empresa incorpora processos para que a inovação seja algo sistemático, ou ela estará sempre agindo na retaguarda, com produtos com menor valor agregado, e consequentemente menos competitivos.

De acordo com Serafim (2011), o sucesso do passado não basta para vencer no futuro. Ter grandes inventores e pioneiros entre seus fundadores ou gestores geniais em algum momento da sua história pode inspirar funcionários, gerar valores importantes, estabelecer processos positivos e ambientes adequados para a inovação. Entretanto, o cenário competitivo se modifica a todo instante e a sobrevivência das organizações exige transformações permanentes.

\subsection{Práticas para Inovação}

Segundo Scherer e Carlomagno (2009), a inovação não é uma atividade eventual, ela é um processo que deve ser gerenciado, desde a ideia inicial até a sua implementação. O processo de inovação inicia-se pela geração de novas ideias, o que os autores chamam de idealização; segue para a conceituação, momento no qual é realizado o refinamento do conceito da ideia proposta; passa pela redução das incertezas, fase em que ocorre a experimentação e chega à implementação. Para Scherer e Carlomagno (2006), é nessa etapa que ocorre a concreta transformação das ideias em inovações. Para Pinchot (2004), a inovação ocorre quando existe uma combinação fortuita de uma ideia, um intra-empreendedor e um patrocinador, todos ao mesmo tempo. 


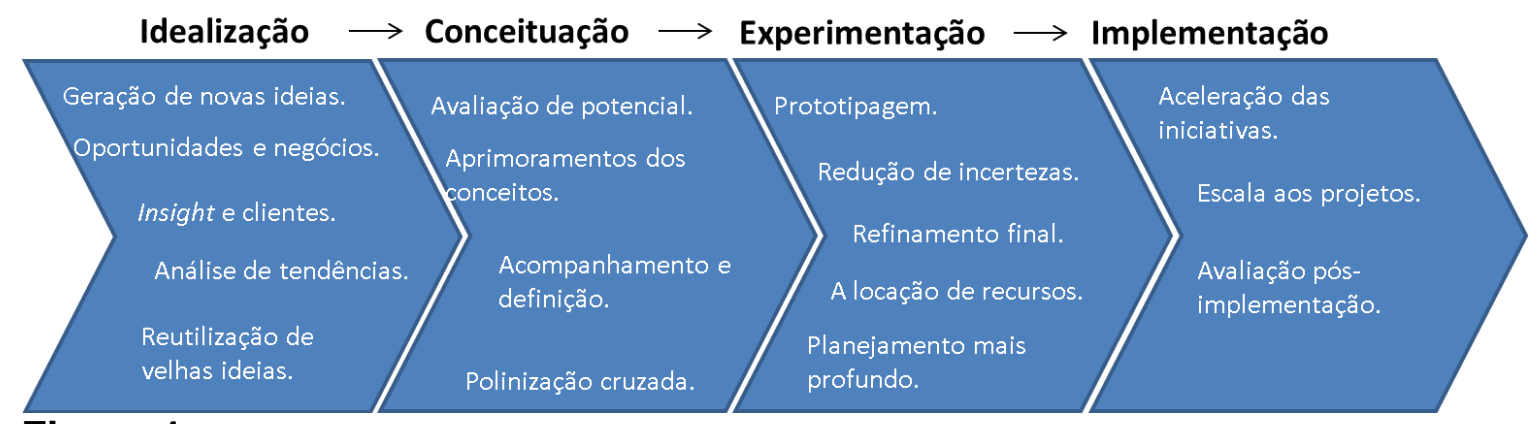

\section{Figura 1}

Processo de inovação

Fonte: Scherer, F. O., \& Carlomagno, M. S. (2009). Gestão da inovação na prática: como aplicar conceitos e ferramentas para alavancar a inovação (p. 23). São Paulo: Atlas.

Segundo Drucker (2013, p. 45), os empreendedores precisam aprender a praticar a inovação sistemática. Isso consiste "na busca deliberada e organizada de mudanças, e na análise sistemática das oportunidades que tais mudanças podem oferecer para a inovação econômica ou social." Para isso, o autor defende o monitoramento de sete fontes.

Essas sete fontes são divididas em dois grupos: (i) ambiente interno; (ii) mudanças fora da empresa, ou seja, no ambiente externo. A Tabela 2 apresenta a relação das fontes de oportunidades com os tipos de ambientes.

\section{Tabela 2}

Inovação sistemática - fontes de oportunidades

\begin{tabular}{l|l}
\hline Ambiente & Fontes de oportunidades \\
\hline \multirow{4}{*}{ Interno } & O inesperado \\
\cline { 2 - 2 } & Incongruências \\
\cline { 2 - 2 } & Necessidade de processo \\
\cline { 2 - 2 } & Estruturas da indústria e do mercado \\
\hline \multirow{3}{*}{ Externo } & Mudanças demográficas \\
\cline { 2 - 2 } & Mudanças em percepção \\
\cline { 2 - 2 } & Conhecimento novo \\
\hline
\end{tabular}

Fonte: Adaptado de Drucker, P. F. (2013). Inovação e espírito empreendedor (entrepreneurship): prática e princípios (C. Malferrari, Trad.). São Paulo: Cengage Learning.

Existe considerável sobreposição entre essas sete fontes de oportunidades, tornando-as indissociáveis. Drucker (2013) atribui o mesmo grau de importância e produtividade a cada uma delas. Porém, as sete fontes requerem análises em separado, pois cada uma delas possui características bem definidas.

Segundo Tidd, Bessant e Pavitt (2008), normalmente a fonte de tecnologia que desestabiliza um setor é externa a ele. Até mesmo empresas que costumam gastar 
tempo e recursos em pesquisa, com a finalidade de estarem à frente dos desenvolvimentos em sua área de atuação, podem descobrir que estão no caminho errado com a entrada de algo que tenha sido desenvolvido em um campo diferente.

A necessidade de processo não se inicia com um evento no meio ambiente, seja interno ou externo. Ela se inicia com o trabalho a ser feito. Está concentrada na tarefa e não concentrada na situação. Aperfeiçoa um processo que já existe, substitui uma ligação que esteja fraca, redesenha um antigo processo fornecendo o "elo que faltava".

Segundo Mattos, Stoffel e Teixeira (2010), a empresa precisa criar um clima organizacional capaz de estimular inovações nos mais variados setores e atividades. Para os autores, as pessoas são o início, meio e fim de qualquer inovação. Ambientes mais abertos e flexíveis atraem pessoas criativas e talentosas. A construção, manutenção e aprimoramento de ambientes voltados para estimular uma cultura voltada para a inovação tem sido um fator crítico de sucesso. A empresa precisa encontrar ferramentas e métodos para a gestão da inovação de acordo com a sua cultura organizacional (Mattos, Stoffel, \& Teixeira, 2010).

Para Tidd, Bessant e Pavitt (2008, p. 85) o desafio consiste em desenvolver formas de gestão da inovação não apenas durante a fase estável, mas sob condições de alta incerteza e velocidade de mudança. Neste cenário, os tipos de comportamentos organizacionais em situação de alta incerteza incluem: agilidade, flexibilidade, habilidade para aprendizagem rápida e ausência de preconceitos sobre a forma como as coisas podem vir a evoluir.

\subsection{Obstáculos para a Inovação}

Na lista de obstáculos para a inovação apontados pela IBGE (2013), constam fatores de natureza econômica (custos, riscos, fontes de financiamento apropriadas), problemas internos à empresa (rigidez organizacional), deficiências técnicas (escassez de serviços técnicos externos adequados, falta de pessoal qualificado), problemas de informação (falta de informações sobre tecnologia e sobre os mercados), problemas com o Sistema Nacional de Inovação (escassas possibilidades de cooperação com outras empresas/ instituições) e problemas de regulação (dificuldade para se adequar a padrões, normas e regulamentações). 
Em muitos setores, segundo Tidd, Bessant e Pavitt (2008), a vantagem advém do conhecimento, porque aquilo que as empresas sabem e possuem é difícil de copiar e exigem que os outros passem por um processo similar de aprendizagem. Alguns conjuntos de conhecimento tornam-se redundantes, e outros precisam ser adquiridos rapidamente, o que reflete na necessidade do gerenciamento estratégico do conhecimento. Para os autores, é provável que o conhecimento relevante para a inovação ocorra fora da empresa, o que exigirá competências para garantir que a transferência de tecnologia possa ser absorvida e explorada rápida e efetivamente.

A empresa precisa substituir ou associar suas competências, que provavelmente não serão mais necessárias no futuro, enquanto adquire e absorve, ao mesmo tempo, novas competências (Tidd, Bessant, \& Pavitt, 2008).

\section{Procedimentos Metodológicos}

Este artigo traz um recorte dos dados coletados no âmbito do projeto "Programa de Incentivos para a Utilização dos Benefícios Fiscais da Lei do Bem". O projeto foi coordenado pelo Instituto Euvaldo Lodi de Santa Catarina (IEL/SC), com o apoio do Ministério da Ciência, Tecnologia e Inovação (MCTI). Uma das etapas do projeto foi o diagnóstico sobre as práticas, obstáculos e desempenho em inovação das empresas optantes pelo regime do lucro real. O presente artigo apresenta os resultados obtidos neste diagnóstico.

Esta pesquisa se caracteriza como descritiva, e de abordagem quantitativa. Como estratégia de pesquisa realizou-se um survey.

\subsection{População e Amostra}

A população deste estudo são todas as indústrias com matriz em Santa Catarina optantes pelo regime tributário do Lucro Real. Para acessar as informações de contato das indústrias utilizou-se o Microsoft $\Theta^{\circledR}$ Dynamics CRM, software de gestão de relacionamento de clientes utilizado pela Federação das Indústrias de Santa Catarina-FIESC. No período de coleta dos dados - entre os meses de julho de 2015 e março de 2016 - havia no estado 2.346 (N) indústrias optantes pelo regime tributário do lucro real cadastradas no sistema.

Todas as indústrias receberam convite, seja por e-mail ou contato telefônico, para participar do estudo. Os convites foram endereçados aos responsáveis pelas áreas contábil, financeira, engenharia, desenvolvimento ou marketing. Ao final do 
período de coleta de dados, 100 (cem) empresas haviam respondido o questionário. A título de análise, caso esta amostra fosse aleatória, teríamos uma margem de erro de $10 \%$, e nível de confiança de $95 \%$. No entanto, como a coleta dos dados dependeu do interesse da empresa em participar, não foi possível realizar uma amostragem puramente probabilística, ou seja, com amostra aleatória. O que se tem, neste estudo, é uma amostra intencional representativa.

\subsection{Instrumento de coleta de dados}

Para a coleta de dados foi desenvolvido um questionário baseado nos instrumentos utilizados por ABDI (2015), Souza e Ruthes (2013) e IBGE (2013). O instrumento teve como objetivo mensurar as práticas de inovação utilizadas pela empresa, assim como identificar quais obstáculos para o processo de inovação são diretamente percebidos pelos respondentes.

O questionário possui 47 perguntas fechadas e uma pergunta aberta. As questões estruturadas estão divididas aleatoriamente em de múltipla escolha e escalas nominais. Neste artigo são apresentados os resultados das questões que avaliam as práticas da empresa, desempenho em produtos/processos e as barreiras para inovação. Essas questões estão estruturadas em escala Likert.

$\mathrm{Na}$ Tabela 3 apresentam as dimensões avaliadas neste estudo com suas respectivas variáveis.

\section{Tabela 3}

Dimensões de análise

\section{Práticas para inovação}

PR1. Os líderes entendem que atingir um equilíbrio entre a necessidade de estabilidade e de ruptura é a chave para a gestão bem sucedida da inovação.

PR2. Até que ponto a sua empresa promove um ambiente que encoraja a criatividade?

PR3. A empresa mantêm-se informada sobre as novas matérias-primas e/ou materiais.

PR4. A empresa mantêm-se informada sobre novos processos produtivos.

PR5. A empresa procura estabelecer um relacionamento de parceria e cooperação com fornecedores que tenham potencial inovador.

PR6. A empresa busca estabelecer uma relação com os seus concorrentes que possibilite a troca de experiências - erros e acertos.

PR7. A empresa realiza benchmarking visando diagnosticar as "melhores práticas" em seu setor de atuação.

PR8. A empresa busca os laboratórios e instituições de Ciência e Tecnologia alinhados ao seu negócio.

PR9. A empresa utiliza banco de competência, tanto interno quanto externo, para os projetos de inovação. 


\section{Desempenho em Inovação}

DP1. Os projetos de inovação proporcionam novas competências para a empresa.

DP2. Os projetos de inovação superam fronteiras científicas ou tecnológicas.

DP3. Os projetos de inovação possuem aspectos fora do escopo de atividade da empresa.

DP4. Os projetos de inovação tem potencial de ser objeto de patente.

DP5. Os projetos de inovação utilizam conhecimentos já existentes, buscando aprofundá-los para resolver problemas específicos.

DP6. Em relação aos produtos (bens ou serviços) inseridos no mercado nos últimos 5 anos mencione com que frequência eles são novos para o mercado nacional, mas já existentes no mercado internacional.

DP7. Em relação aos produtos (bens ou serviços) inseridos no mercado nos últimos 5 anos mencione com que frequência eles são novos para o mercado internacional.

DP8. Em relação aos processos inseridos na empresa nos últimos 5 anos mencione com que frequência eles são novos para o mercado nacional, mas já existentes no mercado internacional.

DP9. Em relação aos processos inseridos na empresa nos últimos 5 anos mencione com que frequência eles são novos para o mercado internacional.

\section{Barreiras para a inovação}

BR1. Riscos econômicos.

BR2. Custos da inovação.

BR3. Escassez de fontes apropriadas de financiamento.

BR4. Desconhecimento dos editais de fomento e linhas de crédito.

BR5. Carência de pessoal qualificado para a elaboração de projetos para captação de recursos.

BR6. Rigidez organizacional.

BR7. Falta de pessoal qualificado para inovação.

BR8. Falta de informação sobre tecnologias.

BR9. Falta de informação sobre mercados.

BR10. Escassas possibilidades de cooperação com outras instituições.

BR11. Dificuldade para se enquadrar a padrões, normas e regulamentações.

BR12. Fraca resposta dos consumidores quanto aos novos produtos.

BR13. Escassez de serviços técnicos externos adequados.

BR14. Falta de materiais e equipamentos.

Fonte: Adaptado de ABDI (2015), Souza e Ruthes (2013) e PINTEC (2011).

O questionário desenvolvido foi para o autopreenchimento, e a coleta de dados foi realizada de forma online por meio da ferramenta Survey Monkey. A consistência de todas as respostas foi verificada pela equipe de pesquisadores logo após o total preenchimento do questionário. Caso fossem verificadas inconsistências nas respostas, os entrevistados eram recontatados a fim de verificar a validade da resposta.

\subsection{Procedimentos para a Análise dos Dados}

Tendo-se em vista que o principal objetivo deste estudo era identificar as principais práticas e entraves para a inovação e relacioná-los com o desempenho obtido em inovação, utilizou-se para a análise dos dados estatística bi e multivariada. 
$\mathrm{Na}$ Tabela 4 apresenta-se as técnicas utilizadas com seus respectivos objetivos e a sequência adotada. Todos os testes estatísticos foram realizados utilizando o software IBM SPSS $\AA$.

\section{Tabela 4}

Técnicas de análise utilizadas

\begin{tabular}{c|l|l}
\hline Etapa & Técnica & Objetivo \\
\hline 1 & Análise Fatorial & $\begin{array}{l}\text { Analisar a relação entre as variáveis observadas e resumi- } \\
\text { las em componentes/fatores. }\end{array}$ \\
\hline 2 & Alpha de Cronbach & Verificar a consistência dos fatores. \\
\hline 3 & Estatística descritiva & $\begin{array}{l}\text { Construir índices de práticas, desempenho e barreiras e } \\
\text { descrever seus resultados. }\end{array}$ \\
\hline 4 & Correlação & $\begin{array}{l}\text { Relacionar os índices de práticas, desempenho e barreiras } \\
\text { para a inovação. }\end{array}$ \\
\hline
\end{tabular}

Fonte: Autores (2017)

De acordo com Loesch e Hoeltgebaum (2005), o propósito essencial da Análise Fatorial é descrever os relacionamentos de covariância entre um conjunto de variáveis e resumi-las em um conjunto altamente correlacionado entre si, e que possua correlações relativamente pequenas com variáveis de outro grupo. Ao avaliar os dados, a análise fatorial obtém dimensões latentes que os descrevem em um número menor de conceitos do que as variáveis individuais originais (Hair et al, 2006, p. 91)

Como as três dimensões analisadas (práticas, desempenho e barreiras) possuem muitas variáveis, utilizou-se a análise fatorial para reduzi-las a um menor número para, na sequência, construir índices. O método de extração dos fatores foi por componentes principais e a rotação deles foi feita pelo método ortogonal de Varimax.

Utilizou-se o Alfa de Cronbach para medir a confiabilidade e a consistência interna do conjunto de variáveis que compõem cada fator resultante da análise fatorial. O valor mínimo aceitável para o alfa é 0,70; abaixo desse valor a consistência interna da escala utilizada é considerada baixa. Em contrapartida, o valor máximo esperado é 0,90; acima deste valor, pode-se considerar que há redundância ou duplicação, ou seja, vários itens estão medindo exatamente o mesmo elemento de um constructo; portanto, os itens redundantes devem ser eliminados (Streiner, 2003). 
A partir da confirmação da confiabilidade e consistência do conjunto de variáveis agrupadas nos fatores da análise fatorial, foram construídos índices visando mensurar a atuação de cada empresa nos fatores obtidos.

Os índices foram construídos a partir do aproveitamento obtido com a somatória de pontos em cada uma das variáveis que compõem cada fator. Os resultados dos índices foram parametrizados entre zero e 100 para permitir a comparabilidade entre os todos os índices criados.

Para análise dos índices foi utilizada estatística descritiva. Para tanto, utilizouse o gráfico Box Plot para ilustrar graficamente cinco medidas estatísticas: valor mínimo, valor máximo, mediana, primeiro e terceiro quartil.

Para avaliar a relação existente entre os índices obtidos, utilizou-se o coeficiente correlação linear de Pearson. Este coeficiente, normalmente representado por $p$, assume apenas valores entre -1 e +1 . O sinal indica a direção, se a correlação é positiva ou negativa, e o tamanho da variável indica a força da correlação, valores de p 0.6 para mais ou para menos indicam uma forte correlação (Barbetta, 2002).

\section{Resultados}

\subsection{Perfil da amostra}

Todas as empresas que participaram do estudo são optantes pelo sistema de tributação do Lucro Real, e atuam em diferentes segmentos da economia: setor alimentício; máquinas e equipamentos; tecnologia da informação e comunicação; têxtil; produtos químicos - plásticos; e construção civil. Quanto ao porte das empresas, utilizou-se a classificação adotada pelo BNDES que categoriza a empresa conforme o faturamento. Na tabela 5 , observa-se que empresas de todos os portes fazerem parte da amostra pesquisada, no entanto, as de médio porte são maioria $(41 \%)$.

\section{Tabela 5}

Porte da empresa com base em seu faturamento anual (classificação BNDES)

\begin{tabular}{l|c}
\hline & Porcentual \\
\hline Microempresa (menor ou igual a $\mathrm{R} \$ 2,4$ milhões) & 4 \\
\hline Pequena empresa (maior que $\mathrm{R} \$ 2,4$ e menor ou igual a $\mathrm{R} \$ 16$ milhões) & 19 \\
\hline Média empresa (maior do que $\mathrm{R} \$ 16$ e menor ou igual a $\mathrm{R} \$ 90$ milhões) & 41 \\
\hline Média grande empresa (menor que $\mathrm{R} \$ 90$ milhões e menor ou igual a $\mathrm{R} \$ 300$ milhões) & 26 \\
\hline Grande empresa (maior que $\mathrm{R} \$ 300$ milhões) & 8 \\
\hline Não informado & 2 \\
\hline
\end{tabular}


Fonte: Dados primários - Mapeamento sobre práticas e barreiras para a inovação - n: 100.

Apesar de as empresas pesquisadas realizarem atividades de PD\&I, verificase que pouco mais da metade (54\%) das empresas não possuem uma área formalizada de PD\&I - Tabela 6.

Os processos de inovação devem permear por toda a organização. As atividades não devem ficar restritas a uma área. No entanto, para a gestão da inovação, é preciso monitorar indicadores, acompanhar ações, etc. A área de PD\&I é responsável por acompanhar esses processos e propor melhorias. Dessa forma, a ausência de uma área formalizada dificulta a sistematização da gestão da inovação.

\section{Tabela 6}

Área de Pesquisa, Desenvolvimento e Inovação formalizada

\begin{tabular}{lc} 
& Porcentagem \\
\hline Sim & 44 \\
Não & 54 \\
Não informado & 2 \\
Total & 100 \\
\hline Fonte: Dados primários - Mapeamento sobre práticas e barreiras para a inovação - n: 100 &
\end{tabular}

\subsection{Redução das dimensões de análise em componentes}

Para a análise dos resultados, o primeiro procedimento estatístico realizado foi a Análise Fatorial. Este teste foi realizado nas três dimensões de análise (Práticas para Inovação; Desempenho em Inovação; e Barreiras para Inovação). Inicialmente, a fim de verificar a adequação da aplicação da análise fatorial foram empregados o teste de Kaiser-Meyer-Olkin (KMO) e o teste de esferacidade de Bartlet.

Segundo Figueiredo Filho e Silva Júnior (2010) o teste de KMO e esferacidade de Bartlet indicam o grau de ajuste dos dados à análise fatorial.

No teste $\mathrm{KMO}$, quanto mais próximos os valores estiverem de 1 indicam que o método de análise fatorial é perfeitamente adequado para o tratamento dos dados. O segundo teste, de esferacidade de Bartlet, é baseado na distribuição estatística do QuiQuadrado e testa a hipótese de que a matriz de correlação é uma matriz de identidade, isto é, não há correlação entre as variáveis. Valores com significância $>0,1$ indicam que os dados não são adequados para o método em questão. Valores menores que o indicado permitem rejeitar a hipótese nula. 
$\mathrm{Na}$ Tabela 7 encontram-se os resultados obtidos com os testes de verificação de adequação da análise fatorial nas três dimensões analisadas.

\section{Tabela 7}

Resultados teste de aderência análise fatorial

\begin{tabular}{l|l|c|c|c}
\hline \multicolumn{2}{l|}{} & Práticas & Barreiras & Desempenho \\
\hline $\begin{array}{l}\text { Medida Kaiser-Meyer-Olkin de adequação de } \\
\text { amostragem. }\end{array}$ &, 827 &, 833 &, 770 \\
\hline \multirow{3}{*}{\begin{tabular}{l} 
Teste de esfericidade de Bartlett \\
\cline { 2 - 5 }
\end{tabular}} & Qui-quadrado aprox. & 179,842 & 339,052 & 236,661 \\
\cline { 2 - 5 } & df & 28 & 55 & 36 \\
\cline { 2 - 5 } & Sig. & 0 & 0 & 0 \\
\hline
\end{tabular}

Fonte: Dados primários - Mapeamento sobre práticas e barreiras para a inovação - n: 100 .

Os valores obtidos nos testes permitem concluir que a análise fatorial é aderente às dimensões de análise. Nos três casos a medida KMO foi superior a 0,7 e a significância do teste de Bartlet é igual a 0, o que confirma a adequação do método de análise fatorial para o tratamento dos dados em questão.

Nas Tabelas 8, 9 e 10 constam os resultados obtidos com as análises fatoriais realizadas em cada uma das dimensões do estudo. Nas tabelas constam as comunilidades obtidas (o valor mínimo aceitável por variável é de 0,5), as cargas fatoriais em cada componente extraído da análise fatorial e o resultado do teste de confiabilidade Alpha de Cronbach para cada componente.

Na Dimensão Práticas para Inovação, foram obtidos dois componentes que explicam cerca de $57,2 \%$ da variabilidade total da dimensão. A variável "PR8 A empresa busca os laboratórios e instituições de Ciência e Tecnologia alinhadas ao seu negócio" foi extraída da análise fatorial, pois obteve comunalidade inferior a 0,5.

O Alpha de Cronbach realizado nas duas componentes originadas na análise fatorial foram significativos (carga superior a 0,6 ). O que confirma a consistência dos componentes e a redução da dimensão em dois subgrupos. As variáveis que compõem o Componente 1 dizem respeito a práticas para aquisição de informações e relacionamentos.

Já o segundo componente reúne variáveis relacionadas com o ambiente interno e cultura da organização, tais como: promoção de um ambiente que encoraje a criatividade dos colaboradores; perfil das lideranças; e utilização de competências internas e externas para os projetos de inovação. 


\section{Tabela 8}

Resultados da análise fatorial para a dimensão Práticas para Inovação e teste de confiabilidade Alpha de Cronbach

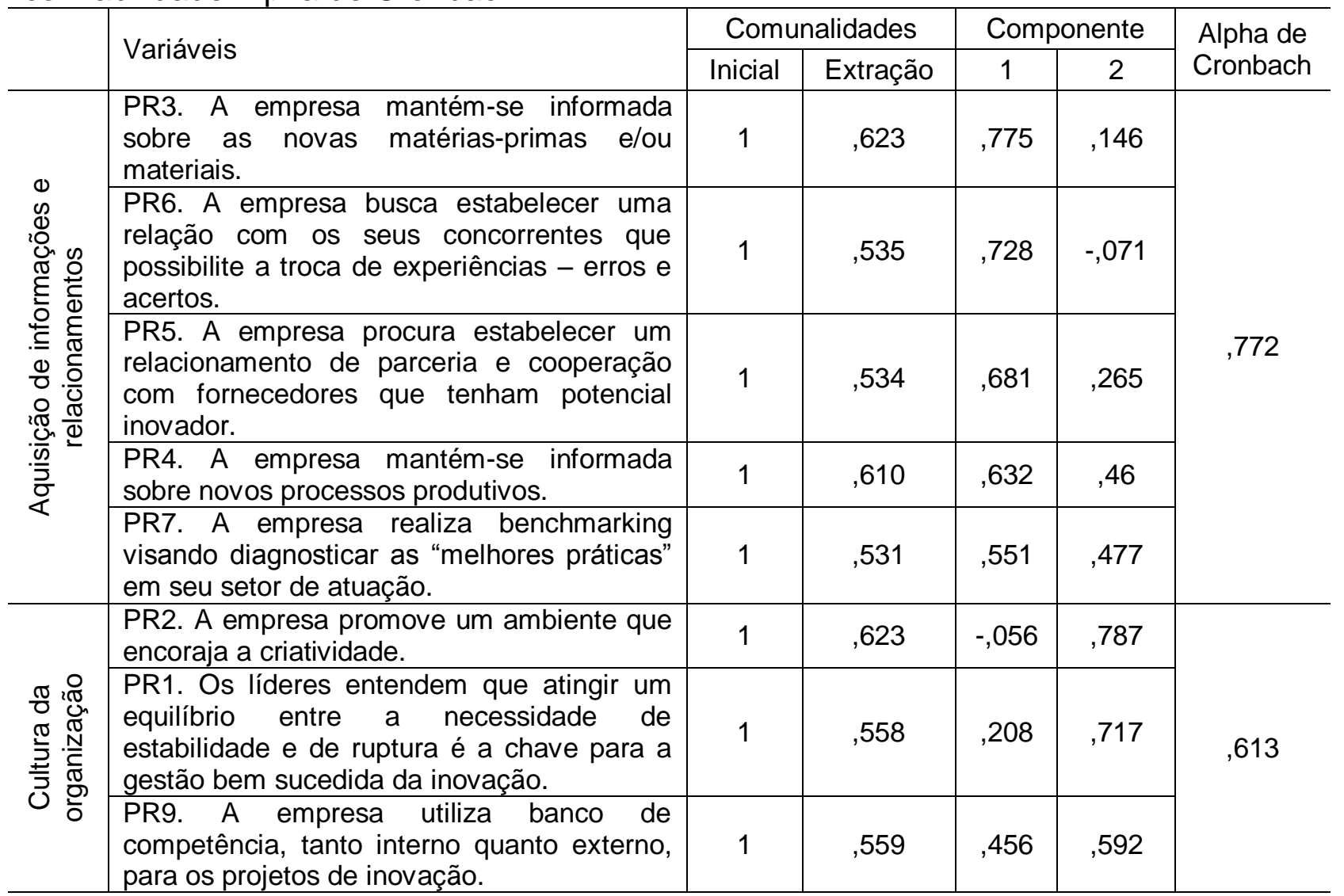

Método de extração: Análise do Componente principal.

Método de rotação: Varimax com normalização de Kaiser.

a Rotação convergida em 3 iterações.

Fonte: Dados primários - Mapeamento sobre práticas e barreiras para a inovação - n: 100.

Ziviani e Ferreira (2013) analisaram os fatores que prejudicam a inovação no setor elétrico no Brasil e os 13 fatores analisados pelos autores foram resumidos em um único fator. Neste estudo, na dimensão Barreiras para Inovação (Tabela 3) a análise fatorial extraiu três componentes que explicam $63,5 \%$ da variação dos dados da dimensão. Das 14 variáveis analisadas somente 11 delas foram validadas no modelo, pois apresentam comunalidade acima de 0,5.

O primeiro componente reúne o maior número de variáveis, são seis ao todo. Essas variáveis dizem respeito, principalmente, às questões de carência de recursos.

O segundo componente diz respeito às questões econômicas e riscos associados, tais como os custos da inovação e a escassez de fontes apropriadas de financiamento. O terceiro componente é formado por duas variáveis, que apesar de 
estarem altamente correlacionadas e possuírem Alpha de Cronbach satisfatórios, são questões que mensuram itens distintos - "BR10. Escassez de fontes apropriadas de cooperação com outras instituições" e "BR4. Desconhecimento dos editais de fomento e linhas de crédito". Dessa forma, essas questões não serão incluídas nos índices de análises.

\section{Tabela 9}

Resultados análise fatorial dimensão Barreiras para Inovação e teste de confiabilidade Alpha de Cronbach

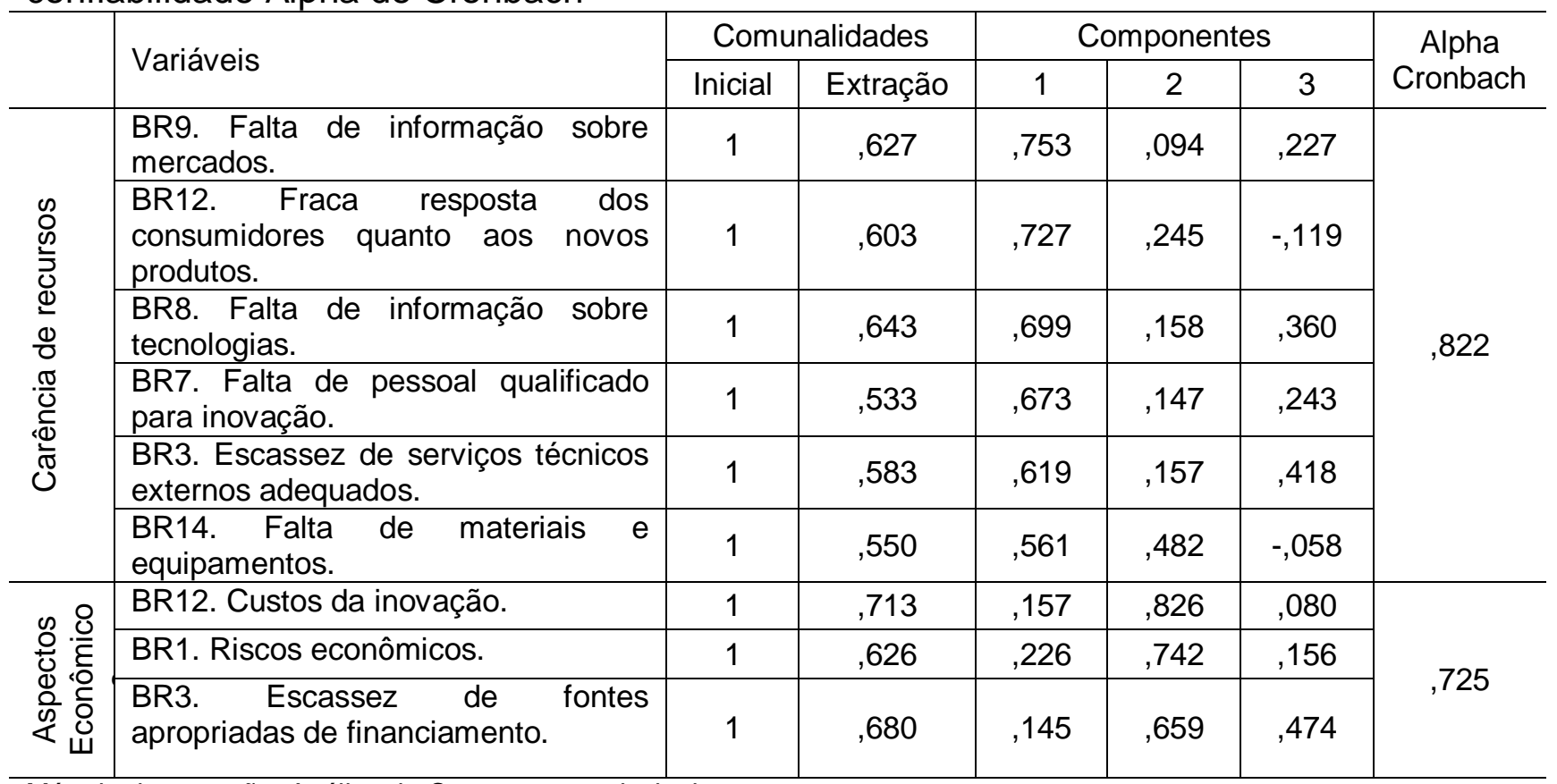

Método de extração: Análise do Componente principal.

Método de rotação: Varimax com normalização de Kaiser.

a. Rotação convergida em 6 iterações.

Fonte: Dados primários - Mapeamento sobre práticas e barreiras para a inovação - n: 100 .

Para analisar o desempenho em inovação foram utilizadas nove variáveis, e todas foram mantidas na análise fatorial, pois possuem comunalidade acima de 0,5 (Tabela 10). O conjunto de variáveis foi dividido em três componentes que explicam $66,4 \%$ da variância das questões que compõem a dimensão.

As variáveis que mensuram o que os projetos de inovação proporcionam para a empresa foram reunidos no primeiro componente. Essas variáveis não mensuram o quanto os novos produtos ou processos foram inovadores para o mercado nacional ou internacional. Questões como estas estão reunidas nos componentes dois e três. Os resultados do Alpha de Cronbach confirmam a consistência da dimensão. 


\section{Tabela 10}

Resultados análise fatorial dimensão Desempenho em Inovação e teste de confiabilidade Alpha de Cronbach

\begin{tabular}{|c|c|c|c|c|c|c|c|}
\hline & \multirow[t]{2}{*}{ Variáveis } & \multicolumn{2}{|c|}{ Comunalidades } & \multicolumn{3}{|c|}{ Componente } & \multirow{2}{*}{$\begin{array}{l}\text { Alpha de } \\
\text { Cronbach }\end{array}$} \\
\hline & & Inicial & Extração & 1 & 2 & 3 & \\
\hline \multirow{5}{*}{ 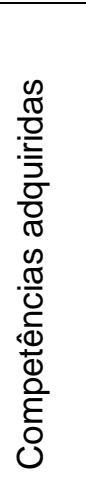 } & $\begin{array}{l}\text { DP2. Superam fronteiras científicas } \\
\text { ou tecnológicas. }\end{array}$ & 1 & ,613 & 750 & 194 & 113 & \multirow{5}{*}{,758 } \\
\hline & $\begin{array}{l}\text { DP5. Utilizam conhecimentos já } \\
\text { existentes, buscando aprofundá-los } \\
\text { para resolver problemas } \\
\text { específicos. }\end{array}$ & 1 & ,507 & 699 &,- 129 & ,035 & \\
\hline & $\begin{array}{l}\text { DP1. Proporcionam novas } \\
\text { competências para a empresa. }\end{array}$ & 1 & ,569 & ,686 & 299 & ,098 & \\
\hline & $\begin{array}{l}\text { DP4. Tem potencial de ser objeto } \\
\text { de patente. }\end{array}$ & 1 & ,525 & 646 & ,302 & ,129 & \\
\hline & $\begin{array}{l}\text { DP3. Possuem aspectos fora do } \\
\text { escopo de atividade da empresa. }\end{array}$ & 1 & ,522 & 538 & ,261 & 405 & \\
\hline \multirow{2}{*}{ 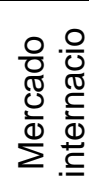 } & $\begin{array}{l}\text { DP7. Novos produtos para } 0 \\
\text { mercado internacional. }\end{array}$ & 1 & ,841 & 211 & 886 & 103 & \multirow{2}{*}{,873 } \\
\hline & $\begin{array}{l}\text { DP9. Novos processos para } 0 \\
\text { mercado internacional. }\end{array}$ & 1 & ,869 & 164 & ,902 & 167 & \\
\hline \multirow{2}{*}{ 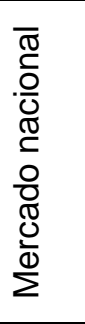 } & 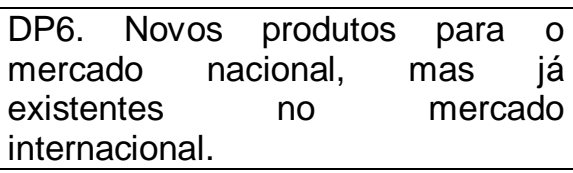 & 1 & ,787 & 192 &,- 023 & 866 & \multirow{2}{*}{,720 } \\
\hline & $\begin{array}{lcc}\text { DP8. Novos processos para } & \text { o } \\
\text { mercado nacional, } & \text { mas já } \\
\text { existentes } & \text { no } & \text { mercado } \\
\text { internacional. } & & \\
\end{array}$ & 1 & ,737 & 043 & 269 & ,814 & \\
\hline
\end{tabular}

Método de extração: Análise do Componente principal.

Método de rotação: Varimax com normalização de Kaiser.

a Rotação convergida em 4 iterações.

Fonte: Dados primários - Mapeamento sobre práticas e barreiras para a inovação - n: 100.

\subsection{Construção e Análise dos Índices de Práticas, Desempenho e Barreiras para a Inovação}

Com o intuito de verificar a relação existente entre as práticas para inovação, barreiras percebidas e desempenho, foram construídos índices a partir dos componentes obtidos nas análises fatoriais. Como as variáveis foram medidas por uma escala de Likert, com variação de pesos entre 0 e 4 , os índices foram construídos com a somatória de pontos obtidos no conjunto de variáveis que compõem cada componente resultante da análise fatorial. Para que fosse possível comparar os resultados obtidos em cada índice os mesmos foram parametrizados entre 0 e 100. 
Na Tabela 11 constam a quantidade de variáveis obtidas em cada índice, a pontuação original máxima obtida (antes de serem parametrizados) e o nome atribuído ao índice.

\section{Tabela 11}

Índices desenvolvidos

\begin{tabular}{l|c|c|c|l}
\hline Abreviação & $\begin{array}{c}\text { Qtdd } \\
\text { variáveis }\end{array}$ & $\begin{array}{c}\text { Pontuação } \\
\text { máxima }\end{array}$ & $\mathrm{N}^{*}$ & Nome do Índice \\
\hline I.PR & 8 & 32 & 71 & Índice Práticas para Inovação \\
\hline I.PR.1 & 5 & 20 & 71 & Aquisição de Informações e Relacionamentos \\
\hline I.PR.2 & 3 & 12 & 71 & Cultura da Organização \\
\hline I.DP & 9 & 36 & 71 & Indice Desempenho em Inovação \\
\hline I.DP.1 & 5 & 20 & 71 & Competências Adquiridas \\
\hline I.DP.2 & 2 & 8 & 71 & Mercado Internacional \\
\hline I.DP.3 & 2 & 8 & 71 & Mercado Nacional \\
\hline I.BR.1 & 6 & 24 & 95 & Carência de Recursos \\
\hline I.BR.2 & 3 & 12 & 95 & Econômico
\end{tabular}

* Nas situações em que pelo menos uma das variáveis possuía valor ausente, a observação foi desconsiderada da análise.

Fonte: Dados primários - Mapeamento sobre práticas e barreiras para a inovação - n: 100 .

A Figura 2 ilustra a extensão dos índices desenvolvidos, incluindo a mediada, valor máximo e mínimo, e os percentis 25 e 75 . A reta ilustra o alcance de todos os dados (o menor ponto de dados encontra-se na extremidade inferior da reta no eixo vertical, e o maior ponto na extremidade superior). As caixas correspondem aos quartis Q1 e Q3. A divisão horizontal na caixa corresponde à mediana do índice. Os círculos correspondem aos outliers - observações que apresentam um grande afastamento das restantes, que possuem valores 1,5 vezes superior ao $3^{\circ}$ quartil ou 1,5 vezes inferior ao $1^{\circ}$ quartil.

Conforme se pode observar no Gráfico 1, de modo geral, as empresas tiveram baixa atuação nos índices pesquisados. Dos 10 índices desenvolvidos, em oito deles $75 \%$ das empresas pesquisadas possuem menos de $60 \%$ de aproveitamento. Os índices de desempenho foram os que apresentaram os resultados mais baixos. $O$ índice que avalia a introdução de novidade no mercado internacional (I.DP2) foi o que obteve pior desempenho. 


\section{Figura 2}

Box Plot com índices de práticas para inovação, desempenho e barreiras

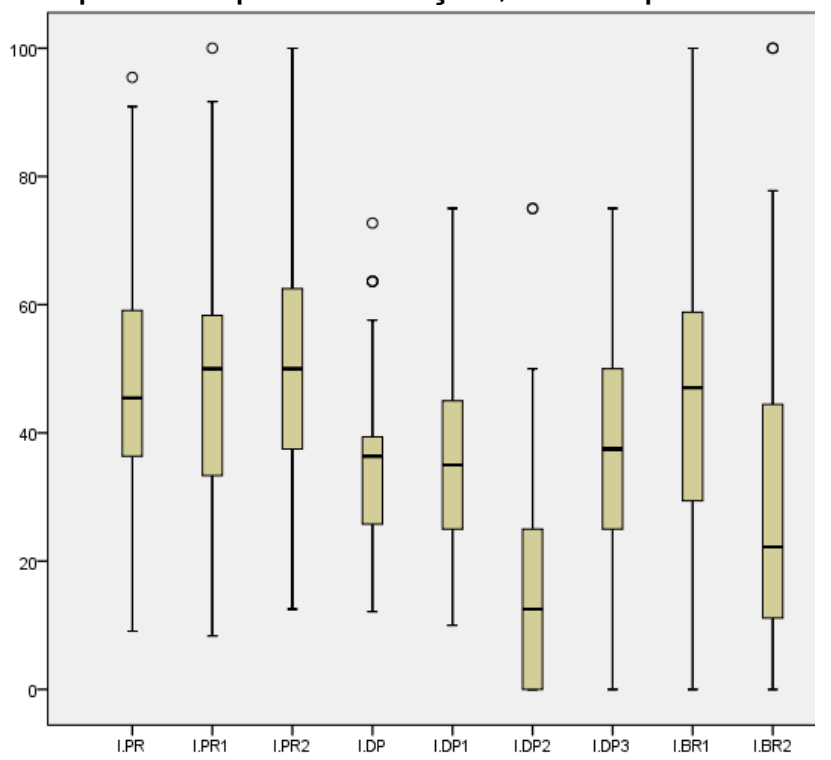

Fonte: Dados primários - Mapeamento sobre práticas e barreiras para a inovação - n: 100

\subsection{Relação entre os índices de práticas, desempenho e barreiras para a inovação}

A fim de diagnosticar a relação existente os índices de práticas para inovação, barreiras e obstáculos utilizou-se o Coeficiente de Correlação de Pearson $(p)$. A Tabela 12 apresenta a matriz identidade obtida incluindo os valores de $p$, significância e o tamanho da amostra. Nas situações em que pelo menos uma das variáveis possuía valor ausente, a observação foi desconsiderada da análise.

Existe correlação positiva significativa entre os índices de práticas para inovação e desempenho. O índice de práticas que envolve aspectos da cultura organizacional (I.PR.2) está fortemente correlacionado com os índices de competências adquiridas (I.DP.1) e introdução de novos produtos ou processos no mercado internacional (I.DP.2). Aquisição de informações e relacionamentos (I.PR.1) também está fortemente relacionado com esses dois índices, porém em um grau um pouco menor.

O índice que mensura o desempenho da empresa na introdução de novos produtos e processos no mercado nacional não está correlacionado com nenhum dos outros índices desenvolvidos neste estudo. 
Não há correlação entre o índice que mensura as práticas adotadas para a aquisição de informações e relacionamentos (I.PR.1) com o índice que mensura as barreiras para a inovação associadas com a escassez de informações e carência técnica (I.BR.1). Uma das hipóteses que se poderia levantar era a de que empresas com elevado índice de práticas em I.PR.1 não observariam as variáveis contidas no índice I.BR.1 como um obstáculo para a inovação (correlação inversa ou negativa). Mas esta hipótese não se confirmou: tanto empresas que adotam práticas para a busca de informações sobre mercados, tecnologias e pessoal para PD\&I, quanto aquelas que não adotam estas práticas observam a carência de informações nesse sentido como um obstáculo para a inovação.

Os índices que possuem correlação inversa, porém pouco significativa, com a percepção quanto às barreiras para a inovação são os que mensuram o desempenho obtido com os projetos de inovação (I.DP), (I.DP.1). As empresas que já colhem frutos com os projetos de inovação possuem uma percepção menos negativa quanto aos obstáculos mensurados neste estudo.

\section{Tabela 12}

Matriz identidade de Correlação de Pearson entre os índices resultantes das análises fatoriais

\begin{tabular}{|c|c|c|c|c|c|c|c|c|c|c|}
\hline & & I.PR & I.PR.1 & I.PR.2 & I.DP & I.DP.1 & I.DP.2 & I.DP.3 & I.BR.1 & I.BR.2 \\
\hline \multirow{4}{*}{ I.PR } & $p$ & 1 & & & & & & & & \\
\hline & Sig. & 0 & & & & & & & & \\
\hline & $\mathrm{N}$ & 71 & & & & & & & & \\
\hline & $p$ &, $891^{* *}$ & 1 & & & & & & & \\
\hline \multirow[t]{3}{*}{ I.PR.1 } & Sig. & 0 & 0 & & & & & & & \\
\hline & $\mathrm{N}$ & 71 & 71 & & & & & & & \\
\hline & $p$ &, $809^{* *}$ &, $506^{\star *}$ & 1 & & & & & & \\
\hline \multirow[t]{2}{*}{ I.PR.2 } & Sig. & 0 & 0 & 0 & & & & & & \\
\hline & $\mathrm{N}$ & 71 & 71 & 71 & & & & & & \\
\hline \multirow{4}{*}{ I.DP } & $p$ &, $606^{\star *}$ &, $480^{\star \star}$ &, $556^{\star \star}$ & 1 & & & & & \\
\hline & Sig. & 0 & 0 & 0 & 0 & & & & & \\
\hline & $\mathrm{N}$ & 71 & 71 & 71 & 100 & & & & & \\
\hline & $p$ &, $571^{* *}$ &, $448^{\star *}$ &, $507^{\star *}$ &, $847^{* *}$ & 1 & & & & \\
\hline \multirow[t]{3}{*}{ I.DP.1 } & Sig. & 0 & 0 & 0 & 0 & 0 & & & & \\
\hline & $\mathrm{N}$ & 71 & 71 & 71 & 100 & 100 & & & & \\
\hline & $p$ &, $501^{\star *}$ &, $414^{\star \star}$ &, $526^{* *}$ &, $646^{\star *}$ &, $437^{* *}$ & 1 & & & \\
\hline \multirow[t]{3}{*}{ I.DP.2 } & Sig. & 0 & 0 & 0 & 0 & 0 & 0 & & & \\
\hline & $\mathrm{N}$ & 71 & 71 & 71 & 100 & 100 & 100 & & & \\
\hline & $p$ & 220 & ,167 & ,168 &, $589^{* *}$ &, $394^{* *}$ &, $295^{\star *}$ & 1 & & \\
\hline \multirow[t]{2}{*}{ I.DP.3 } & Sig. & ,065 & ,165 &, 16 & 0 & 0 & ,003 & 0 & & \\
\hline & $\mathrm{N}$ & 71 & 71 & 71 & 100 & 100 & 100 & 100 & & \\
\hline
\end{tabular}




\begin{tabular}{l|l|ccc|cccc|cc}
\hline & $p$ &, 048 &,- 007 &, 145 &,$- 234^{*}$ &,$- 245^{*}$ &,- 017 &,- 039 & 1 & \\
I.BR.1 & Sig. &, 689 &, 953 &, 229 &, 023 &, 017 &, 872 &, 705 & 0 & \\
& $\mathrm{~N}$ & 71 & 71 & 71 & 95 & 95 & 95 & 95 & 95 & \\
\cline { 2 - 10 } & $\mathrm{p}$ &,- 065 &,- 118 &, 100 &,$- 268^{* *}$ &,$- 251^{*}$ &, 023 &,- 147 &, $616^{* *}$ & 1 \\
I.BR.2 & Sig. &, 588 &, 326 &, 406 &, 009 &, 014 &, 822 &, 154 & 0 & 0 \\
& $\mathrm{~N}$ & 71 & 71 & 71 & 95 & 95 & 95 & 95 & 95 & 95 \\
\hline
\end{tabular}

p: Correlações de Pearson $\quad{ }^{*} p<0,05{ }^{* \star} p<0,01$

Fonte: Dados primários - Mapeamento sobre práticas e barreiras para a inovação - n: 100.

\section{Considerações Finais}

Este artigo teve como objetivo apresentar as principais barreiras para a inovação identificadas em um grupo de 100 indústrias com matriz no estado de Santa Catarina optantes pelo regime tributário do Lucro Real e relacioná-las com algumas práticas por elas adotadas.

A principal pergunta de pesquisa deste estudo era se as organizações possuem práticas que estimulam a inovação inseridas em suas rotinas. Ao responder essa pergunta, os empresários mencionaram os aspectos relacionados ao ambiente externo como os principais entraves para a inovação, como se a inovação fosse um processo que se iniciasse primeiro no ambiente externo, para depois ser inserido no ambiente interno da organização. Não estamos dizendo que fatores externos não afetam o ambiente organizacional. Muito pelo contrário. O ecossistema de inovação no qual a organização está inserida afeta diretamente no seu comportamento. Porém, o sistema só existe quando as instituições atuam em rede, conectadas com o mesmo fim.

Como conclusão, verificou-se que os empresários mencionaram questões de natureza econômica como os principais entraves para a inovação. Ou seja, na visão deles, o problema para o fraco desempenho em inovação diz respeito apenas aos fatores externos. Porém, práticas que poderiam ser incorporadas às rotinas das empresas que resultam diretamente no desempenho em inovação são pouco exploradas.

Os dados deste artigo comprovaram que a performance em inovação é resultado das práticas adotadas pela empresa. Os fatores determinantes para o desempenho em inovação vão desde a busca por novas matérias primas e processos produtivos, até o relacionamento de cooperação com fornecedores e concorrentes. Todas essas práticas tornam-se mais fluidas e efetivas para a 
organização quando a mesma possui uma área formalizada para cuidar dos assuntos relacionados à Pesquisa, Desenvolvimento e Inovação - PD\&l.

Observa-se que os obstáculos para a inovação vão além dos que estão explícitos para as empresas. A adoção de práticas que as auxiliem na conexão com os incentivos oferecidos pelo ambiente externo ainda são incipientes. Como exemplo, menciona-se os incentivos ficais da Lei 11.196/2005, conhecida como Lei do Bem. Menos de 1\% das empresas com enquadramento fiscal fazem uso desses incentivos. O maior obstáculo apontado pelos pesquisados está na carência de linhas de fomento para a inovação. No entanto, a maior parte das linhas de crédito para inovação apoiam projetos cujo escopo ultrapasse a fronteira tecnológica. Além do potencial inovador dos projetos, para receber tais benefícios são analisadas a estrutura de gestão da inovação, bem como o gerenciamento contábil e financeiro das empresas. E, na prática, o que se observa é que poucas empresas se estruturam para a inovação criando áreas específicas para tratar deste assunto. Além disso, os projetos raramente superam fronteiras tecnológicas ou possuem aspectos fora do escopo de desenvolvimento da empresa. As inovações se restringem a melhorias de processos ou inovações incrementais, envolvendo pouco risco tecnológico. As práticas para inovação utilizadas pelas empresas resultam em produtos ou processos com pouca novidade para 0 mercado nacional $\mathrm{e}$ internacional. Isso se confirma nos dados apresentados neste artigo.

O maior obstáculo para a inovação ocorre devido à falta de visão dos empresários sobre as ações que de fato são impulsionadoras de inovação. Os resultados obtidos neste estudo comprovam que o desempenho obtido em inovação é resultado das práticas adotadas pelas empresas. Ou seja, para se conectar com os incentivos oferecidos pelo ambiente externo, a empresa precisa inicialmente mudar as suas práticas. Aspectos relacionados à cultura organizacional estão fortemente correlacionados com a introdução de novos produtos ou processos no mercado.

Recomenda-se, para estudos futuros, a replicação do estudo em uma amostra maior ou em setores específicos, a fim de validar os componentes extraídos e índices desenvolvidos.

\section{Referências}


ABDI - Agência Brasileira de Desenvolvimento Industrial (2014). Sondagem de inovação da $A B D I: 4^{\circ}$ trimestre.

Barbetta, P. A. (2002). Estatística aplicada às ciências sociais (5a ed.). Florianópolis: UFSC.

Drucker, P. F. (2013). Inovação e espírito empreendedor (entrepreneurship): prática e princípios (C. Malferrari, Trad.). São Paulo: Cengage Learning.

D'Este, P., lammarino, S., Savona, M., \& Tunzelmannc, N. V. (2012). What hampers innovation? Revealed barriers versus deterring barriers. Research Policy 41, $482-488$.

Feldens, M. A., Maccari, E. A., \& Garcez, M. P. (2012). Barriers for production innovation in small and medium technology-based firms in Brazil. $B B R-$ Brazilian Business Review, 9(3), 1 - 22.

Figueiredo Filho, D. B., \& Silva Júnior, J. A. da (2010). Visão além do alcance: uma introdução à análise fatorial. Opin. Publica, 16(1). DOI:

http://dx.doi.org/10.1590/S0104-62762010000100007

GEM - Global Entrepreurship Monitor (2013). Empreendedorismo no Brasil. Curitiba: IBQP.

Hair Jr., Black, W. C., Babin, B. J., Anderson, R. E. E., \& Tatham, R. L. (2006). Multivariate Data Analysis (6a ed.). Upper Saddle River, NJ: Pearson Prentice Hall.

IBGE - Instituto Brasileiro de Geografia e Estatística (2013). PINTEC: Pesquisa de Inovação 2011. IBGE: Rio de Janeiro.

Kühl, M. R., \& Cunha, J. C. da (2013). Obstacles to implementation of innovations in Brazil: how different companies perceive their importance. BBR-Brazilian Business Review, 10(2), $1-24$.

Lei n. 10.973, de 02 de Dezembro de 2004. Dispõe sobre incentivos à inovação e à pesquisa científica e tecnológica no ambiente produtivo e dá outras providências. Diário Oficial da União, Poder Executivo, Brasília, DF, 03 de Dezembro de 2004. Disponível em: <https://www.planalto.gov.br/ccivil_03/_Ato20042006/2004/Lei/L10.973.htm>. Acesso em: 14 de Maio de 2014.

Loesch, C., \& Hoeltgebaum, M. (2005). Métodos estatísticos multivariados aplicados à economia de empresas. Blumenau: Nova Letra.

Mattos, J. F., Stoffel, H. R., \& Teixeira, R. de A. (2010). Mobilização Empresarial pela Inovação: cartilha, gestão da inovação. Confederação Nacional das Indústrias: Brasília.

Oslo - OECD (2006). Manual de Oslo: Proposta de Diretrizes para a Coleta e Interpretação de dados sobre Inovação Tecnológica. Terceira Edição.

Pinchot, G. P. (2004). Intra-empreendedorismo na prática: um guia de inovação nos negócios. Rio de Janeiro: Elsevier.

Porter, M. E. (1999). Competição: estratégias competitivas essenciais (C. da Cunha Trad.). Rio de Janeiro: Campus. 
Scherer, F. O., \& Carlomagno, M. S. (2009). Gestão da inovação na prática: como aplicar conceitos e ferramentas para alavancar a inovação. São Paulo: Atlas.

Schumpeter, J. A. (1988). Teoria do desenvolvimento econômico: uma investigação sobre lucros, capital, crédito, juro e o ciclo econômico (3a ed., M. S. Possas Trad.). São Paulo: Nova Cultural.

Serafim, L. (2011). O poder da inovação: como alavancar a inovação na sua empresa. São Paulo: Saraiva.

Souza, M. de, \& Ruthes, S. (2013). Bússola da inovação: relatórios técnicos setoriais. Curitiba: SENAI/PR.

Streiner, D. L. (2003). Being inconsistent about consistency: when coefficient alpha does and doesn't matter. Journal of Personality Assessment, 80, 217-222.

Terra, J. C. (2000). Gestão do conhecimento: o grande desafio empresarial, uma abordagem baseada no aprendizado e na criatividade. São Paulo: Negócio Editora.

Tidd, J., Bessant, J., \& Pavitt, K. (2008). Gestão da Inovação, (3a ed.). Porto Alegre: Bookman.

Ziviani, F., \& Ferreira, M. A. T. (2013). Barreiras e Obstáculos à Inovação no Setor Elétrico Brasileiro: Desafios e Oportunidades. Revista Gestão \& Tecnologia, 13(3), 222-246. 